\title{
Optical Injection Dynamics of VCSEL Frequency Combs
}

\author{
Yaya Doumbia ${ }^{1,2}$, Delphine Wolfersberger ${ }^{1,2}$, Krassimir Panajotov $^{3,4}$, Marc Sciamanna $^{1,2}$ \\ 1. Chaire Photonique, CentraleSupélec, 2 Rue Edouard Belin 57070 Metz, France \\ 2. Université de Lorraine, CentraleSupélec, LMOPS, 2 Rue Edouard Belin 57070 Metz, France \\ 3. Brussels Photonics Group (B-PHOT), Vrije Universiteit Brussel, Brussels, Belgium \\ 4. Institute of Solid-State Physics, Bulgarian Academy of Sciences, Sofia, Bulgaria
}

Recent works have shown the possibility to control through optical injection frequency combs generated by gain switching in Vertical-Cavity Surface-Emitting Lasers (VCSELs) [1]. The opposite situation, in which VCSEL is subject to injection of an optical frequency comb (OFC) deserves close attention and has not been addressed so far. In this work, we analyze the nonlinear polarization dynamics of a single-mode VCSEL with optical injection. Here, we select and reveal several bifurcations scenarios leading to generation of a two polarization OFC.

Figure 1 shows a sequence of bifurcation when the VCSEL is subject to parallel optical injection with three frequency comb lines with comb spacing $\Omega=4 \mathrm{GHz}$ and detuning $\Delta v=-0.9 \mathrm{GHz}$. The polarization of injection is parallel to the one of the VCSEL. Fig.1 (a) shows time-periodic dynamics corresponding to an OFC but that significantly extends the number of comb lines, as we also evidenced in a DFB laser [2,3].
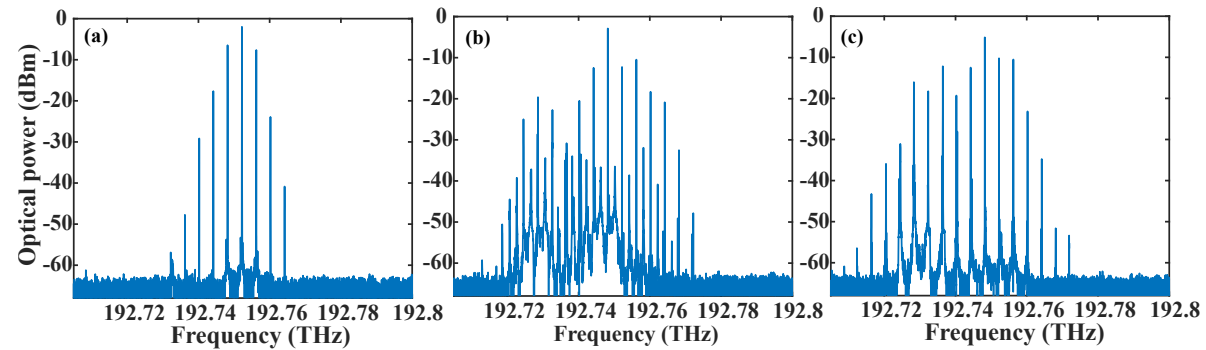

Fig. 1 Bifurcation scenario leading to two polarization frequency comb for $\Omega=4 \mathrm{GHz}$ and $\Delta v=-0.9 \mathrm{GHz}$. (a) polarized OFC at $P_{i n j}=0.3 \mu \mathrm{W}$, (b) two polarization harmonics OFC at $P_{i n j}=141 \mu \mathrm{W}$, and (c) two polarization OFC at $P_{i n j}=210 \mu \mathrm{W}$.

When increasing the injected power, the VCSEL bifurcates from an OFC in the dominant polarization to an unstable OFC with excitation of the depressed polarization mode. For some injection parameters, a harmonics OFC can be observed that extend over the two polarization modes as shown in Fig 1 (b). When further increasing the injected power, the VCSEL output shows again a time-periodic dynamics corresponding to an OFC (Fig.1(c)) at the same repetition rate as the injected comb. Polarization-resolved measurement confirm that these time-periodic dynamics are the contribution of two linear orthogonal polarization time-periodic dynamics. Our experiment data demonstrated the possibility to optimize the Carrier to Noise Ratio and bandwidth of the comb by tuning both the injection parameters and the polarization of injected comb lines.

Numerical simulations is performed to analyze the phase dynamics of the two polarization frequency comb. Interestingly, we found that each orthogonal frequency combs is phase locked. Our theoretical results also predict the possibility of controlling the comb spacing through the generation of comb harmonics until reaching new comb solutions with a repetition rate smaller than $100 \mathrm{MHz}$.

In summary, experiments and numerical simulations tell us that injection of an OFC into a VCSEL allows sustaining polarization dynamics leading to very wideband frequency combs extending up 13 times the bandwidth of injected comb. These results may satisfy the demand of the growing capacity through polarization division multiplexing for optical data communications [4], and others applications such dual spectroscopy.

Funding Chaire Photonique: Ministère de l'Enseignement supérieur, de la Recherche et de l'Innovation; Région Grand Est; Département Moselle; European Regional Development Fund (ERDF); Metz Metropole; Airbus GDI Simulation; Fondation CentraleSupélec; Fonds Wetenschappelijk Onderzoek (FWO) (G0E5819N).

\section{References}

[1] A. Quirce, C. De Dios, A. Valle, and P. Acedo, "Vcsel-based optical frequency combs expansion induced by polarized optical injection," IEEE Journal of Selected Topics in Quantum Electronics 25, 1-9 (2018).

[2] Y. Doumbia, T. Malica, D. Wolfersberger, K. Panajotov, and M. Sciamanna, "Nonlinear dynamics of a laser diode with an injection of an optical frequency comb," Optics Express 28, 30379-30390 (2020)

[3] Y. Doumbia, T. Malica, D. Wolfersberger, K. Panajotov, and M. Sciamanna, "Optical injection dynamics of frequency combs," Optics Letters 45, 435-438 (2020).

[4]D. Ivanovich, S. B. Powell, V. Gruev, and R. D. Chamberlain, "Polarization division multiplexing for optical data communications," in Optical Inter-connects XVIII, Vol. 10538 (International Society for Optics and Photonics,2018) p. 105381D. 